\title{
The splitting number can be smaller than the matrix chaos number
}

\author{
by \\ Heike Mildenberger (Vienna) and Saharon Shelah (Jerusalem)
}

\begin{abstract}
Let $\chi$ be the minimum cardinality of a subset of ${ }^{\omega} 2$ that cannot be made convergent by multiplication with a single Toeplitz matrix. By an application of a creature forcing we show that $\mathfrak{s}<\chi$ is consistent. We thus answer a question by Vojtáš. We give two kinds of models for the strict inequality. The first is the combination of an $\aleph_{2}$-iteration of some proper forcing with adding $\aleph_{1}$ random reals. The second kind of models is obtained by adding $\delta$ random reals to a model of $\mathrm{MA}_{<\kappa}$ for some $\delta \in\left[\aleph_{1}, \kappa\right)$. It was a conjecture of Blass that $\mathfrak{s}=\aleph_{1}<\chi=\kappa$ holds in such a model. For the analysis of the second model we again use the creature forcing from the first model.
\end{abstract}

0. Introduction. We consider products of $\omega \times \omega$ matrices $A=\left(a_{i, j}\right)_{i, j<\omega}$ of reals and functions from $\omega$ to 2 or to some bounded interval of the reals. We define

$$
A \lim f:=\lim _{i \rightarrow \infty} \sum_{j=0}^{\infty}\left(a_{i, j} \cdot f(j)\right) .
$$

Toeplitz (cf. [2]) showed: $A \lim$ is an extension of the ordinary limit iff $A$ is a regular matrix, i.e. iff $(\exists m)(\forall i)\left(\sum_{j=0}^{\infty}\left|a_{i, j}\right|<m\right)$ and $\lim _{i \rightarrow \infty} \sum_{j=0}^{\infty} a_{i, j}=1$ and $(\forall j)\left(\lim _{i \rightarrow \infty} a_{i, j}=0\right)$. Regular matrices are also called Toeplitz matrices.

We are interested in whether for many $f$ 's simultaneously there is one $A$ such that all $A \lim f$ exist, and formulate our question in terms of cardinal characteristics.

Let $\ell^{\infty}$ denote the set of bounded real sequences, and let $\mathbb{M}$ denote the set of all Toeplitz matrices. Vojtáš [10] defined for $\mathbb{A} \subseteq \mathbb{M}$ the chaos relations $\chi_{\mathbb{A}, \infty}$ and their norms $\left\|\chi_{\mathbb{A}, \infty}\right\|$ as follows:

2000 Mathematics Subject Classification: 03E15, 03E17, 03E35.

The first author was supported by a Minerva fellowship.

The second author's research was partially supported by the "Israel Science Foundation", founded by the Israel Academy of Science and Humanities. This is the second author's work number 753 . 


$$
\begin{aligned}
\chi_{\mathbb{A}, \infty}=\left\{(A, f): A \in \mathbb{A} \wedge f \in \ell^{\infty} \wedge A \lim f \text { does not exist }\right\}, \\
\left\|\chi_{\mathbb{A}, \infty}\right\|=\min \left\{|\mathcal{F}|: \mathcal{F} \subseteq \ell^{\infty} \wedge\right. \\
(\forall A \in \mathbb{A})(\exists f \in \mathcal{F})(A \lim f \text { does not exist })\} .
\end{aligned}
$$

By replacing $\ell^{\infty}$ by ${ }^{\omega} 2$, the set of $\omega$-sequences with values in 2 , we get the variations $\chi_{\mathbb{A}, 2}$. In $[6]$ we showed that for the cardinals we are interested in, $\omega_{2}$ and $\ell^{\infty}$ give the same result. From now on we shall work with ${ }^{\omega} 2$.

Vojtáš (cf. [11]) also gave some bounds valid for any $\mathbb{A}$ that contains at least all matrices which have exactly one non-zero entry in each line:

$$
\mathfrak{s} \leq\left\|\chi_{\mathbb{A}, 2}\right\| \leq \mathfrak{b} \cdot \mathfrak{s} .
$$

We write $\chi$ for $\left\|\chi_{\mathbb{M}, 2}\right\|$.

In [6] we showed that $\chi<\mathfrak{b} \cdot \mathfrak{s}$ is consistent relative to ZFC. Here, we show the complementary consistency result, that $\mathfrak{s}<\chi$ is consistent. We get the convergence with positive matrices.

Now we recall the definitions of the cardinal characteristics $\mathfrak{b}$ and $\mathfrak{s}$ involved: The order of eventual dominance $\leq^{*}$ is defined as follows: For $f, g \in \omega^{\omega} \omega$ we say $f \leq^{*} g$ if there is $k \in \omega$ such that for all $n \geq k$ we have $f(n) \leq g(n)$.

The unbounding number $\mathfrak{b}$ is the smallest size of a subset $\mathcal{B} \subseteq{ }^{\omega} \omega$ such that for each $f \in \omega_{\omega}$ there is some $b \in \mathcal{B}$ such that $b \mathbb{Z}^{*} f$. The splitting number $\mathfrak{s}$ is the smallest size of a subset $\mathcal{S} \subseteq[\omega]^{\omega}$ such that for each $X \in[\omega]^{\omega}$ there is some $S \in \mathcal{S}$ such that $X \cap S$ and $X \backslash S$ are both infinite. The latter is expressed as " $S$ splits $X$ ", and $\mathcal{S}$ is called a splitting family. For more information on these cardinal characteristics, we refer the reader to the survey articles $[1,3,9]$.

If $A \lim f$ exists, then so $\operatorname{does} A^{\prime} \lim f$ for any $A^{\prime}$ that is obtained from $A$ by erasing rows and moving the remaining (infinitely many) rows together. We may further change $A^{\prime}$ by keeping only finitely many non-zero entries in each row, so that the neglected ones have a negligible absolute sum, and then possibly multiplying the remaining ones so that they again sum up to 1 . Hence, after possibly a further deletion of lines we may restrict the set of Toeplitz matrices to linear Toeplitz matrices. A matrix is linear iff each column $j$ has at most one entry $a_{i, j} \neq 0$ and for $j<j^{\prime}$ the $i$ with $a_{i, j} \neq 0$ is smaller than or equal to the $i$ with $a_{i, j^{\prime}} \neq 0$ if both exist, in picture:

$$
\left(\begin{array}{ccccccc}
c_{0}(0) & \ldots c_{0}\left(m_{\mathrm{up}}\left(c_{0}\right)-1\right) & 0 & \ldots & 0 & 0 & \ldots \\
0 & \ldots 0 & c_{1}\left(m_{\mathrm{dn}}\left(c_{1}\right)\right) & \ldots & c_{1}\left(m_{\mathrm{up}}\left(c_{1}\right)-1\right) & 0 & \ldots \\
0 & \ldots 0 & 0 & \ldots & 0 & c_{2}\left(m_{\mathrm{dn}}\left(c_{2}\right)\right) & \ldots \\
\vdots & & & & & &
\end{array}\right)
$$

Linear matrices can be naturally (as in the picture) read as $\left\langle c_{i}\right\rangle_{i<\omega}$ where $c_{i}:\left[m_{\mathrm{dn}}\left(c_{i}\right), m_{\mathrm{up}}\left(c_{i}\right)\right) \rightarrow[0,1], c_{i}(j)=a_{i, j}$, give the finitely many non-zero 
entries in row $i$, and $m_{\mathrm{up}}\left(c_{i-1}\right)=m_{\mathrm{dn}}\left(c_{i}\right)$. The $c_{i}$ 's are special instances of the weak creatures in the sense of [7]. In the next two sections we shall show: The $c_{i}$ 's coming from the trunks of the conditions in the generic filter of our forcing $Q$ give matrices that make, after multiplication, members of $\omega_{2}$ from the ground model and members of $\omega_{2}$ of any random extension convergent.

Now that we have used the word "creature" several times, we should explain it. Roughly speaking, creatures are certain partial functions. Countably many of them are put into an arrangement which serves as one forcing condition. Stronger conditions are obtained by composing (usually finitely many) partial functions and changing the arrangement in a certain way. We need the exact definitions only at one point in our work, when we want to cite some result on properness from [7]. For this purpose, we have to verify that our forcing is an instance of a creature forcing built from a finitary creating pair, such that the forcing conditions fulfil some conditions on the growth of the norms of their building blocks. We shall explain these notions in the next section.

1. A creature forcing. In this section, we give a self-contained description of the creature forcing $Q$ which is the main tool for building the two kinds of models in the next section. Moreover, we explain the connections and give the references to [7], so that the reader can identify it as a special case of an extensive framework.

Definition 1.1. We define a forcing notion $Q$. We say that $p \in Q$ if $p=\left(n,\left\langle c_{i}\right\rangle_{i<\omega}\right)$ (or $p=\left(n^{p},\left\langle c_{i}^{p}\right\rangle_{i<\omega}\right)$ when we want to avoid confusion comparing two elements of $Q)$ and

(1) $n<\omega$

(2) for each $i$ there are natural numbers $m_{\mathrm{dn}}\left(c_{i}\right)<m_{\mathrm{up}}\left(c_{i}\right)$ such that $c_{i}:\left[m_{\mathrm{dn}}\left(c_{i}\right), m_{\mathrm{up}}\left(c_{i}\right)\right) \rightarrow[0,1]$,

(3) $(\forall i<\omega)\left(\forall k \in \operatorname{dom}\left(c_{i}\right)\right)\left(c_{i}(k) \cdot k ! \in \mathbb{Z}\right)$,

(4) $(\forall i<\omega)\left(\sum_{k \in \operatorname{dom}\left(c_{i}\right)} c_{i}(k)=1\right)$,

(5) $m_{\mathrm{up}}\left(c_{i}\right)=m_{\mathrm{dn}}\left(c_{i+1}\right)$.

We let $p \leq q$ (" $q$ is stronger than $p$ ") if

(6) $n^{p} \leq n^{q}$,

(7) $\left(\forall 0 \leq k<n^{p}\right)\left(c_{k}^{p}=c_{k}^{q}\right)$,

(8) there exists an increasing sequence $\left\langle k_{t}\right\rangle_{t \geq n^{p}}$ of natural numbers such that for each $t \geq n^{p}$ there exist a non-empty set $u_{t} \subseteq\left[k_{t}, k_{t+1}\right)$ and positive rationals $\left\{d_{l}: l \in u_{t}\right\}$ such that $\sum_{l \in u_{t}} d_{l}=1$ and $c_{t}^{q}=\sum_{l \in u_{t}} d_{l} c_{l}^{p}$.

Observe that if $p \leq q$ then for each $t \geq n^{p}$ we have $m_{\mathrm{dn}}\left(c_{t}^{q}\right)=m_{\mathrm{dn}}\left(c_{k_{t}}^{p}\right)$ and $m_{\mathrm{up}}\left(c_{t}^{q}\right)=m_{\mathrm{up}}\left(c_{k_{t+1}-1}^{p}\right)$. 
We write $p \leq_{i} q$ if $n^{p}=n^{q}$ and $c_{j}^{q}=c_{j}^{p}$ for $j<n^{p}+i$.

REMARK 1.2. The notation we used in 1.1 is natural to describe our forcing in a compact manner. However, it does not coincide with the notation given for the general framework in [7]. Here is a translation: We write $\left(\left\langle c_{i}^{p}\right\rangle_{i<n^{p}},\left\langle c_{i}^{p}\right\rangle_{i \geq n^{p}}\right)$ instead of $\left(n^{p},\left\langle c_{i}^{p}\right\rangle_{i<\omega}\right)$, which contains the same information. Then we write

$$
\left(\left\langle c_{i}^{p}\right\rangle_{i<n^{p}},\left\langle c_{i}^{p}\right\rangle_{i \geq n^{p}}\right)=\left(w^{p},\left\langle t_{i}^{p}\right\rangle_{i<\omega}\right)
$$

i.e. we shift the indices.

Now we want to show that $Q$ is proper. This follows from the work in [7] once we have verified that $Q$, in the form $(*)$, fulfils all the conditions on forcing notions in $[7,2.1 .6]$.

We claim that there is a set $K$ of creatures with respect to a finitary $\mathbf{H}$ and a subcomposition function $\Sigma$ such that $(K, \Sigma)$ is finitary $[7,1.1 .3(2)]$ and such that our forcing is $Q_{s, \infty}^{*}(K, \Sigma)$ in the notation of [7].

We take $\mathbf{H}: \omega \rightarrow V$ such that $\mathbf{H}(i)=\{0,1 / i !, 2 / i !, \ldots,(i !-1) / i !, 1\}$ for $i \in \omega$. As usual we write $\triangleleft$ for the proper initial segment relation. The set of all weak creatures with respect to $\mathbf{H}$ is the set of all $t=(\operatorname{nor}(t), \operatorname{val}(t), \operatorname{dis}(t))$ such that $\operatorname{nor}(t) \in \mathbb{R}, \operatorname{nor}(t)>0$, and $\operatorname{val}(t)$ is a non-empty subset of

$$
\left\{(x, y) \in \bigcup_{m_{0}<m_{1}<\omega}\left[\prod_{i<m_{0}} \mathbf{H}(i) \times \prod_{i<m_{1}} \mathbf{H}(i)\right]: x \triangleleft y\right\} .
$$

$K$ will be a subset of the set of all weak creatures. The function dis is the empty function in our case. There are the following requirements on $t \in K$ : For $i \in \omega, t_{i}$ from $(*)$ is a part of such a $t$ in the following sense: $\operatorname{nor}(t)=m_{\mathrm{dn}}\left(t_{i}\right), \operatorname{range}(\mathbf{v a l}(t))=\left\{t_{i}\right\}$. For $\operatorname{dom}(\mathbf{v a l}(t))$ one can take the maximal set fitting to $\operatorname{val}(t)$, since the range does not depend on the domain in our case. We see that $\mathbf{H}$ and $K$ are finitary in the sense of $[7,1.1 .3(2)]$. Now we take $\Sigma:[K]^{<\omega} \rightarrow \mathcal{P}(K), \Sigma\left(\left\{t_{0}, \ldots, t_{n-1}\right\}\right)=\emptyset$ unless $t_{i}:\left[m_{\mathrm{dn}}\left(t_{i}\right), m_{\mathrm{up}}\left(t_{i}\right)\right) \rightarrow \mathbb{R}, t_{i} \in K$, and $m_{\mathrm{up}}\left(t_{i}\right)=m_{\mathrm{dn}}\left(t_{i+1}\right)$, in which case $\Sigma\left(\left\{t_{0}, \ldots, t_{n-1}\right\}\right)$ is the set of all $t:\left[m_{\mathrm{dn}}\left(t_{0}\right), m_{\mathrm{up}}\left(t_{n-1}\right)\right) \rightarrow \mathbb{R}, t \in K$, such that there are $d_{l}, l \in n$, with $\sum_{l \in n} d_{l}=1$ and $t(m)=d_{l} t_{l}(m)$ for each $l$ and $m \in\left[m_{\mathrm{dn}}\left(t_{l}\right), m_{\mathrm{up}}\left(t_{l}\right)\right)$. Our $\Sigma$ is a subcomposition operation in the sense of $[7,1.1 .4]$. Now some further quite long definitions (the interested reader may look at 1.1.6 to $1.1 .10,1.2 .1$ to 1.2 .6 in [7]) give that our instance $(K, \Sigma)$ is a finitary creating pair and that our $Q$ is $Q_{s, \infty}^{*}(K, \Sigma)$ in Rosłanowski and Shelah's framework. Now their work shows:

LEMma 1.3 ([7, Corollary 2.1.6]). The forcing notion $Q$ is proper.

2. The effect of $Q$ on random reals. Let $G$ be $Q$-generic over $V$. We set $c_{i}^{G}=c_{i}^{q}$ for $q \in G$ and $n^{q}>i$. This is well defined. Let $\underset{\sim}{c_{i}}$ be a 
name for it. Our aim is to show that multiplication by the matrix whose $i$ th row is $c_{i}$ makes any real from the ground model and even any real from a random extension of the ground model convergent. For background information about random reals we refer the reader to [5, §42]. The Lebesgue measure is denoted by $\lambda$. By "adding $\kappa$ random reals" we mean forcing with the measure algebra $R_{\kappa}$ on $2^{\omega \times \kappa}$, that is, adding $\kappa$ random reals at once or "side-by-side" and not successively.

Definition 2.1. (1) Let $\operatorname{may}_{k}(p)=\left\{c_{i}^{r}: p \leq_{k} r, i \geq n^{p}+k\right\}$.

(2) For a function $c: \operatorname{dom}(c) \rightarrow \mathbb{R}$ with finite domain and $\eta \in{ }^{\omega} 2$ let

$$
\operatorname{av}(\eta, c)=\sum_{k \in \operatorname{dom}(c)} c(k) \eta(k) .
$$

Main Lemma 2.2. Assume that

(A) $\eta$ is a random name of a member of ${ }^{\omega} 2, \eta=f(\underset{\sim}{r})$ where $f$ is Borel and $\underset{\sim}{r}$ is a name of the random generic real,

(B) $p \in Q$,

(C) $k^{*}<\omega$.

Then for every $k \geq k^{*}$ there is some $q(k) \in Q$ such that

( $\alpha) p \leq_{k^{*}} q(k)$,

( $\beta$ ) for all $l$, if $k^{*} \leq k<l<\omega$ and $c_{1}, c_{2} \in \operatorname{may}_{l}(q(k))$ then

$$
\frac{1}{l !}>\lambda\left\{r: \frac{3}{2^{k}} \leq\left|\operatorname{av}\left(f(r), c_{1}\right)-\operatorname{av}\left(f(r), c_{0}\right)\right|\right\} .
$$

Proof. For $q \in Q$ and $k, l \in \omega, i \in\left\{0,1, \ldots, 2^{k}\right\}$ we set

$$
\begin{aligned}
\operatorname{err}_{k, i}\left(\sim_{\sim}, c\right) & =\int_{0}^{1}\left|\operatorname{av}(f(r), c)-\frac{i}{2^{k}}\right| d r \\
\mathrm{e}_{k, i}^{l}(\underset{\sim}{\eta}, q) & =\inf \left\{\operatorname{err}_{k, i}(\underset{\sim}{\eta}, c): c \in \operatorname{may}_{l}(q)\right\} .
\end{aligned}
$$

Note that $\operatorname{err}_{k, i}(\underset{\sim}{\eta}, c)$ is a real and no longer a random name. So the infimum is well defined.

Now, if $l_{1}<l_{2}$ then $\operatorname{may}_{l_{1}}(q) \supseteq \operatorname{may}_{l_{2}}(q)$ and hence

$$
\mathrm{e}_{k, i}^{l_{1}}(\underset{\sim}{\eta}, q) \leq \mathrm{e}_{k, i}^{l_{2}}(\underset{\sim}{\eta}, q) \text {. }
$$

So $\left\langle\mathrm{e}_{k, i}^{l}(\eta \sim, q)\right\rangle_{l<\omega}$ is an increasing bounded sequence and

$$
\mathrm{e}_{k, i}^{*}(\underset{\sim}{\eta}, q)=\lim _{l \rightarrow \infty} \mathrm{e}_{k, i}^{l}(\underset{\sim}{\eta}, q)
$$

is well defined. 
We fix $i \leq 2^{k}$, until Subclaim 4, when we start looking at all $i$ together.

SubClaim 1. There is some $q_{1}^{k, i}=q_{1} \geq_{k^{*}} p$ such that for $l \geq k^{*}$,

$$
\mathrm{e}_{k, i}^{*}(\underset{\sim}{\eta}, p)-1 / l \leq \operatorname{err}_{k, i}\left(\underset{\sim}{\eta}, c_{l}^{q_{1}}\right) \leq \mathrm{e}_{k, i}^{*}(\underset{\sim}{\eta}, p)+1 / l .
$$

Moreover, if $m_{\mathrm{dn}}\left(c_{l^{\prime}}^{q_{1}}\right)=m_{\mathrm{dn}}\left(c_{l}^{p}\right)$ then $\mathrm{e}_{k, i}^{l^{\prime}}\left(\eta \sim, q_{1}\right) \geq \mathrm{e}_{k, i}^{*}(\underset{\sim}{\eta}, p)-1 / l$.

Why? We choose $c_{l}^{q_{1}}$ by induction on $l$ : For $l \leq n^{p}+k^{*}$, we take $c_{l}^{q_{1}}=c_{l}^{p}$. Suppose that we have chosen $c_{m}^{q_{1}}$ for $m<l$ and that we are to choose $c_{l}^{q_{1}}$, $l>n^{p}+k^{*}$. We set $\varepsilon=1 / l$. By possibly end-extending $c_{l-1}^{q_{1}}$ by zeroes we may assume that $m_{\mathrm{up}}\left(c_{l-1}^{q_{1}}\right)=m_{\mathrm{up}}\left(c_{l^{\prime}}^{p}\right)$ for an $l^{\prime} \geq l$ so large that for all $l^{\prime \prime} \geq l^{\prime}, \mathrm{e}_{k, i}^{l^{\prime \prime}}(\underset{\sim}{\eta}, p) \geq \mathrm{e}_{k, i}^{*}(\underset{\sim}{\eta}, p)-\varepsilon$. Then we take $c_{l}=c_{l}^{q_{1}} \in \operatorname{may}_{l^{\prime \prime}}(p)$ such that $\operatorname{err}_{k, i}\left(\underset{\sim}{\eta}, c_{l}^{q_{1}}\right) \leq \mathrm{e}_{k, i}^{l^{\prime \prime}}(\underset{\sim}{\eta}, p)+\varepsilon \leq \mathrm{e}_{k, i}^{*}(\underset{\sim}{\eta}, p)+\varepsilon$. On the other hand we have $\operatorname{err}_{k, i}\left(\underset{\sim}{\eta}, c_{l}^{q_{1}}\right) \geq \mathrm{e}_{k, i}^{l^{\prime \prime}}(\underset{\sim}{\eta}, p) \geq \mathrm{e}_{k, i}^{*}(\underset{\sim}{\eta}, p)-\varepsilon$. The fact that this holds also for $l^{\prime} \leq l$ if $m_{\mathrm{dn}}\left(c_{l^{\prime}}^{q_{1}}\right)=m_{\mathrm{dn}}\left(c_{l}^{p}\right)$ yields the "moreover" part.

SubClaim 2. In Claim 1 , if $l \geq k^{*}$ and $q_{1}^{k, i} \leq_{l} q_{2}$ then

$$
\mathrm{e}_{k, i}^{*}(\underset{\sim}{\eta}, q)-1 / l \leq \operatorname{err}_{k, i}\left(\underset{\sim}{\eta}, c_{l}^{q_{2}}\right) \leq \mathrm{e}_{k, i}^{*}(\eta \sim, q)+1 / l .
$$

Why? By the definition it suffices to show:

$(\otimes) \quad$ if $l_{1}<\ldots<l_{t}<\omega$ and $d_{1}, \ldots, d_{t} \geq 0$ and $d_{1}+\ldots+d_{t}=1$, and $c_{l}^{q_{2}}=d_{1} c_{1}^{q_{1}}+\ldots+d_{t} c_{t}^{q_{1}}$, then

$$
\mathrm{e}_{k, i}^{*}\left(\underset{\sim}{\eta}, q_{1}\right)-1 / l \leq \operatorname{err}_{k, i}\left(\underset{\sim}{\eta}, c_{l}^{q_{2}}\right) \leq \mathrm{e}_{k, i}^{*}\left(\underset{\sim}{\eta}, q_{1}\right)+1 / l .
$$

The first inequality holds by the "moreover" part in the previous claim. For the second inequality it suffices to show that

$$
\operatorname{err}_{k, i}(\underset{\sim}{\eta}, c) \leq \sum_{s=1}^{t} d_{s} \operatorname{err}_{k, i}\left(\underset{\sim}{\eta}, c_{s}^{q_{1}}\right)
$$

For this it suffices to see that

$$
\int_{0}^{1}\left(\left|\operatorname{av}(f(r), c)-i / 2^{k}\right|\right) d r \leq \sum_{s=1}^{t} d_{s} \int_{0}^{1}\left(\left|\operatorname{av}\left(f(r), c_{s}^{q_{1}}\right)-i / 2^{k}\right|\right) d r,
$$

and since $d_{s} \geq 0$ and $\sum_{s} d_{s}=1$ we finish by the triangle inequality.

SubClaim 3. Let $q^{k, i}$ be as in Subclaim 2. For all $l$, if $c_{0}, c_{1} \in \operatorname{may}_{l}\left(q_{1}^{k, i}\right)$, then

$$
\frac{2^{k+1}}{l} \geq \lambda\left\{r: \operatorname{av}\left(f(r), c_{0}\right) \geq \frac{i+1}{2^{k}} \wedge \operatorname{av}\left(f(r), c_{1}\right) \leq \frac{i-1}{2^{k}}\right\} .
$$

Why? Consider $c=\frac{1}{2} c_{0}+\frac{1}{2} c_{1} \in \operatorname{may}_{l}\left(q_{1}\right)$. Write

$$
A=\left\{r: \operatorname{av}\left(f(r), c_{0}\right) \geq \frac{i+1}{2^{k}} \wedge \operatorname{av}\left(f(r), c_{1}\right) \leq \frac{i-1}{2^{k}}\right\} .
$$


Then

$$
\begin{aligned}
\frac{2}{l} \geq & \frac{1}{2} \operatorname{err}_{k, i}\left(\underset{\sim}{\eta}, c_{0}\right)+\frac{1}{2} \operatorname{err}_{k, i}\left(\underset{\sim}{\eta}, c_{1}\right)-\operatorname{err}_{k, i}(\underset{\sim}{\eta}, c) \\
= & \int_{0}^{1}\left(\frac{1}{2}\left|\operatorname{av}\left(f(r), c_{0}\right)-\frac{i}{2^{k}}\right|+\frac{1}{2}\left|\operatorname{av}\left(f(r), c_{1}\right)-\frac{i}{2^{k}}\right|\right. \\
& \left.-\mid \operatorname{av}(f(r)), c)-\frac{i}{2^{k}} \mid\right) d r \\
\geq & \int_{A}\left(\frac{1}{2}\left|\operatorname{av}\left(f(r), c_{0}\right)-\frac{i}{2^{k}}\right|+\frac{1}{2}\left|\operatorname{av}\left(f(r), c_{1}\right)-\frac{i}{2^{k}}\right|\right. \\
& \left.-\mid \operatorname{av}(f(r)), c)-\frac{i}{2^{k}} \mid\right) d r \\
\geq & \frac{1}{2^{k}} \lambda(A) .
\end{aligned}
$$

SubClaim 4. For every $q \in Q$ and $k^{*}$ we can find $q^{k}$ such that

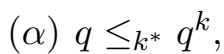

( $\beta)$ if $l \in[k, \omega)$ and $c_{0}, c_{1} \in \operatorname{may}_{l}\left(q^{k}\right)$ and $i \in\left\{1,2, \ldots, 2^{k}-1\right\}$ then

$$
\frac{2^{k+1}}{l}>\lambda\left\{r: \operatorname{av}\left(f(r), c_{0}\right) \geq \frac{i+1}{2^{k}} \wedge \operatorname{av}\left(f(r), c_{1}\right) \leq \frac{i-1}{2^{k}}\right\} \text {. }
$$

$(\gamma)$ This holds also for every $q^{*} \geq q^{k}$.

Why? Repeat Subclaims $1-3$ choosing $q^{k, i}, i=0,1, \ldots, 2^{k}$. We let $q_{0}=q$ and choose $q^{k, i+1}$ which relates to $q^{k, i}$ like $q_{1}$ to $q$.

Now $q^{k}=q^{k, 2^{k}}$ is O.K. Note that according to $(\otimes)$ thinning and averaging can only help.

SubClAim 5. Let $q^{k}$ be as in Subclaim 4. For $l \geq k$ there is $q(k, l)$ $\geq_{l-1} q^{k}$ such that for $c_{0}, c_{1} \in \operatorname{may}_{l}(q(k, l))$,

$$
1 / l !>\lambda\left\{r: 3 / 2^{k} \leq\left|\operatorname{av}\left(f(r), c_{1}\right)-\operatorname{av}\left(f(r), c_{0}\right)\right|\right\} .
$$

Why? The event $3 / 2^{k} \leq\left|\operatorname{av}\left(f(r), c_{1}\right)-\operatorname{av}\left(f(r), c_{0}\right)\right|$ implies that for some $i \in\left\{1,2, \ldots, 2^{k}-1\right\}$ we have

$$
\operatorname{av}\left(f(r), c_{1}\right) \geq \frac{i+1}{2^{k}} \wedge \operatorname{av}\left(f(r), c_{2}\right) \leq \frac{i-1}{2^{k}}
$$

or vice versa. So it is included in the union of $2 \times\left(2^{k}-1\right)$ events, each of measure $\leq 2^{k+1} / l$. Hence it itself has measure $\leq 2^{2 k+2} / l$. By thinning out $q^{k}$ (by moving the former $l$ far out by putting in a lot of zeroes and thus having as new $c_{l}$ 's partial functions that were formerly labelled with a much larger $l$ and thus giving a much smaller quotient according to Subclaim 4) we replace $2^{2 k+2} / l$ by $1 / l$ !

Finally we come to the $q(k)$ from part $(\beta)$ of the lemma: 
SubCLAIM 6. For any $k$ there is $q(k)$ such that $q \leq_{k^{*}} q(k)$ and for any $l \geq k$ and any $c_{0}, c_{1} \in \operatorname{may}_{l}\left(q^{*}\right)$,

$$
1 / l !>\lambda\left\{r: 3 / 2^{k} \leq\left|\operatorname{av}\left(f(r), c_{1}\right)-\operatorname{av}\left(f(r), c_{0}\right)\right|\right\} .
$$

Why? As in the previous claim we choose inductively $q(k, l)$ such that $q_{0}=p$ and $q(k, l+1) \geq_{l} q(k, l)$ and $(q(k, l+1), q(k, l), l)$ are like $(q(k, l), q, l)$ from Subclaim 5 , but for larger and larger $l$. Now

$$
q(k)=\left(n^{p}+k, c_{0}^{p}, \ldots, c_{n^{p}+k}^{p}, c_{n^{p}+k+1}^{q\left(k, n^{p}+k+1\right)}, c_{n^{p}+k+1}^{q\left(k, n^{p}+k+2\right)}, \ldots\right)
$$

is as required in $(\alpha)$ and $(\beta)$ of the conclusion; we have even $q(k) \geq_{k} p$.

Now we use the Main Lemma in an iteration. Any forcing notion $Q^{\prime}$ that preserves $\aleph_{1}$ and $\aleph_{2}$ is suitable. In the application, $Q^{\prime}$ will be an end segment of length $\aleph_{2}$ of a countable support iteration of $Q$.

Conclusion 2.3. Let $Q^{\prime}$ be any notion of forcing. Then we have: $\vdash_{Q}$ "if $\underset{\sim}{\eta} \in V$ is a random name for a real in $V^{Q *{\underset{\sim}{Q}}^{\prime} * R_{\omega}}$ then " $\vdash_{\mathcal{Q}^{\prime} * R_{\omega}}$ $\left\langle\operatorname{av}\left(\underset{\sim}{\eta}, \underset{\sim}{c_{n}}\right): n \in \omega\right\rangle$ converges." "

Proof. Let $q \in Q$ and $\varepsilon>0$ be given. Let $\eta=f(\underset{\sim}{r}), f \in V$, be a random name for a real. We take $k_{0}$ such that $3 / 2^{k_{0}} \tilde{<} \varepsilon$. Then we take $q(k) \geq q$ as in the Main Lemma. We set

$$
A_{k, c_{0}, c_{1}}=\left\{r: 3 / 2^{k}>\left|\operatorname{av}\left(f(r), c_{1}\right)-\operatorname{av}\left(f(r), c_{0}\right)\right|\right\} .
$$

Since $\sum_{l \geq 1} 1 / l !<\infty$, we can apply the Borel-Cantelli lemma and get:

For any sequence $\left\langle c_{l}\right\rangle_{l<\omega}$ such that $c_{l} \in \operatorname{may}_{l}\left(q^{*}(k)\right)$ we have

$$
\lambda\left(\bigcup_{K \in[k, \omega)} \bigcap_{l \geq K} A_{k_{0}, c_{l}, c_{l+1}}\right)=1 .
$$

So $r \in \bigcap_{l \geq K} A_{k, c_{l}, c_{l+1}}$ for some $K \geq k$. So $q(k)$ forces (also in $V[G]$ where $G$ is $Q * Q^{\prime} * R_{\omega}$-generic over $\left.V\right)$ that $\left\langle c_{l}\right\rangle_{l<\omega}$ describes a matrix whose product

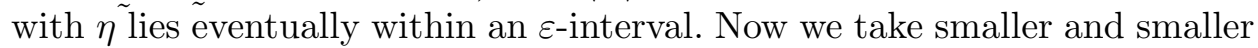
$\varepsilon ' s$ and apply a density argument.

Conclusion 2.4. Let $P_{\omega_{2}}=\left\langle P_{i}, Q_{j}: i \leq \omega_{2}, j<\omega_{2}\right\rangle$ be a countable support iteration of $\underline{\sim}_{i}$, where $Q_{i}$ is $Q$ defined in $V^{P_{i}}$, and let $\underset{\sim}{R_{\omega_{1}}}$ be a $P_{\omega_{2}}$-name of the $\aleph_{1}$-random algebra. Then in $V^{P_{\omega_{2}} * R_{\omega_{1}}}$ we have $\mathfrak{s}=\aleph_{1}$ and $\chi>\aleph_{1}$.

Proof. Dow proves in [4, Lemma 2.3] that $s=\aleph_{1}$ after adding $\aleph_{1}$ or more random reals, over any ground model. In order to show $\chi>\aleph_{1}$, let $\eta_{i}, i<\omega_{1}$, be reals in $V^{P_{\omega_{2}} * R_{\omega_{1}}}$. Over $V^{P_{\omega_{2}}}$, each $\eta_{i}$ has an $R_{\omega_{1}}$-name $\eta_{i}$. Since the random algebra is c.c.c., without loss of generality there are only countably many of the $\aleph_{1}$ random reals mentioned in $\eta_{i}$. Let $\eta_{i}^{\prime}$ be obtained from $\eta_{i}$ by replacing these countably many by the first $\omega$ ones and then acting as if there were just one random real. This is possible because $R_{1}$ 
and $R_{\omega}$ are equivalent forcings. Since the random algebra is c.c.c., the name $\eta_{i}^{\prime}$ can be coded as a single real $r_{i}$ in $V^{P_{\omega_{2}}}$. Now, by $[8$, V.4.4] and by the properness of the $Q_{j}$, this name $r_{i}$ appears at some stage $\alpha\left(\eta_{i}\right)<\aleph_{2}$ in the iteration $P_{\omega_{2}}$. We take the supremum $\alpha$ of all the $\alpha\left(\eta_{i}\right), i<\omega_{1}$. We apply 2.3 with $Q=Q_{\alpha}, Q^{\prime}=\left\langle P_{i}, Q_{j}: \alpha<i \leq \omega_{2}, \alpha<j<\omega_{2}\right\rangle$ and $R_{\omega}$ to the $\eta_{i}^{\prime}$. Thus $Q_{\alpha}$ adds a Toeplitz matrix that makes, by multiplication, all the $\tilde{\eta}_{i}^{\prime}$ convergent. Since Conclusion 2.3 applies to all random algebras simultaneously, this matrix makes also the $\eta_{i}$ convergent.

Definition 2.5. (1) $Q_{\mathrm{pr}}=\left\{p \in Q: n^{p}=0\right\}$ is called the pure part of $Q$.

(2) We write $p \leq^{*} q$ if there are some $w, n$ such that $p \leq\left(w, t_{n}^{q}, t_{n+1}^{q}, \ldots\right)$. So, it is $p \leq q$ up to a finite "mistake".

FACT 2.6. If $\left\langle p_{i}: i\langle\gamma\rangle\right.$ is $\leq^{*}$-increasing in $Q$ and $\mathrm{MA}_{|\gamma|}$ holds, then there is $p \in Q_{\mathrm{pr}}$ such that for all $i<\delta, p_{i} \leq^{*} p$.

Proof. We apply $\mathrm{MA}_{|\gamma|}$ to the following partial order $P$ : Conditions are $(s, F)$ where $s=\left(t_{0}^{p}, \ldots, t_{n}^{p}\right)$ is an initial segment of a condition in $Q_{\mathrm{pr}}$ and $F \subset \gamma$ is a finite set. We let $(s, F) \leq_{P}(t, G)$ iff $s \unlhd t$ and $F \subseteq G$ and $(\forall n \in \lg (t)-\lg (s))(\forall \alpha \in F)\left(n>\left(\right.\right.$ all mistakes between the $\left.p_{\alpha}\right) \rightarrow t_{n} \in$ $\Sigma\left(c_{i}^{p_{\alpha}}: i \in \mathcal{S}(\alpha, n)\right.$ for suitable $\left.\left.\mathcal{S}(\alpha, n)\right)\right)$. This forcing is c.c.c., because conditions with the same first component are compatible and because there are only countably many possibilities for the first component. It is easy to see that for $\alpha<\delta$ the sets $D_{\alpha}=\{(s, F): \alpha \in F\}$ are dense and that for $n \in \omega$ the sets $D^{n}=\{(s, F): \lg (s) \geq n\}$ are dense. Hence if $G$ is generic, then $p=\bigcup\{s:(\exists F)((s, F) \in G)\} \geq^{*} p_{\alpha}$ for all $\alpha$.

ConcLusion 2.7. If $V \models \mathrm{MA}_{\kappa}$ and $\kappa>\delta>\aleph_{0}$, then in $V^{R_{\delta}}$ the matrix number is at least $\kappa$ and the splitting number is $\aleph_{1}$.

Proof. As mentioned, [4] shows the result on the splitting number. For the matrix number, let random names $\eta_{i}, i<\gamma$, be given in $V, \gamma<\kappa$. We fix $\varepsilon>0$ and $K$ as in the proof of 2.3. For $i<\gamma$, we choose $p^{i}=\left\langle c_{k}^{i}\right\rangle_{k<\omega}$ as at the end of the proof of 2.3 for $\eta_{i}$, use Fact 2.6 iteratively $\gamma+1$ times and find a pure condition $p=\left\langle c_{k}\right\rangle_{k<\omega} \geq^{*} p^{i}$ for all $i<\gamma$, that gives the lines of a matrix which brings everything into an $\varepsilon$-range. We denote these $c_{k}$ by $c_{k}=c_{k}(\varepsilon)$. Now by induction we choose $c_{k}: c_{0}=c_{0}(1)$, and $c_{k}=c_{k^{\prime}}\left(1 /\left(k^{\prime}+1\right)\right)$ if $k^{\prime}>k$ is the first $k^{\prime \prime}$ such that $m_{\mathrm{dn}}\left(c_{k^{\prime \prime}}\left(1 /\left(k^{\prime \prime}+1\right)\right)\right)>m_{\mathrm{dn}}\left(c_{k-1}\right)$. The matrix with $c_{k}$ in the $k$ th line acts as desired. (Now $m_{\mathrm{up}}\left(c_{k}\right)>m_{\mathrm{dn}}\left(c_{k+1}\right)$ is possible but this does not harm.)

Acknowledgements. The first author would like to thank Andreas Blass for discussions on the subject and for reading and commenting. 


\section{References}

[1] A. Blass, Combinatorial cardinal characteristics of the continuum, in M. Foreman et al. (eds.), Handbook of Set Theory, Kluwer, to appear.

[2] R. Cooke, Infinite Matrices and Sequence Spaces, MacMillan, 1950.

[3] E. van Douwen, The integers and topology, in: K. Kunen and J. Vaughan (eds.), Handbook of Set-Theoretic Topology, North-Holland, 1984, 111-167.

[4] A. Dow, Tree $\pi$-bases for $\beta \mathbb{N}-\mathbb{N}$, Topology Appl. 33 (1989), 3-19.

[5] T. Jech, Set Theory, Academic Press, 1978.

[6] H. Mildenberger, Convergence and Toeplitz matrices, Fund. Math. 165 (2000), 175189.

[7] A. Rosłanowski and S. Shelah, Norms on possibilities I: Forcing with trees and creatures, Mem. Amer. Math. Soc. 671 (1999).

[8] S. Shelah, Proper Forcing, Lecture Notes in Math. 940, Springer, 1982.

[9] J. E. Vaughan, Small uncountable cardinals and topology, in: J. van Mill and G. Reed (eds.), Open Problems in Topology, Elsevier, 1990, 195-218.

[10] P. Vojtáš, More on set theoretic characteristics of summability by regular (Toeplitz) matrices, Comm. Math. Univ. Carolin. 29 (1988), 97-102.

[11] - Series and Toeplitz matrices (a global implicit approach), Tatra Mountain Math. J. 14 (1998), 269-281.

Institut für formale Logik

Universität Wien

Währinger Str. 25

A-1090 Vienna, Austria

E-mail: heike@logic.univie.ac.at
Institute of Mathematics The Hebrew University of Jerusalem

Givat Ram

91904 Jerusalem, Israel

E-mail: shelah@math.huji.ac.il

Received 28 November 2000;

in revised form 22 May 2001 